\title{
Nontrivial Phase Coupling in Polariton Multiplets
}

\author{
H. Ohadi, ${ }^{1}$ R. L. Gregory, ${ }^{1}$ T. Freegarde, ${ }^{1}$ Y. G. Rubo, ${ }^{2}$ A. V. Kavokin, ${ }^{1,3}$ N. G. Berloff, ${ }^{4,5,}{ }^{*}$ and P. G. Lagoudakis ${ }^{1,4, \dagger}$ \\ ${ }^{1}$ School of Physics and Astronomy, University of Southampton, Southampton, SO17 1BJ, United Kingdom \\ ${ }^{2}$ Instituto de Energías Renovables, Universidad Nacional Autónoma de México, \\ Temixco, Morelos, 62580, Mexico \\ ${ }^{3}$ Spin Optics Laboratory, St-Petersburg State University, 1, Ulianovskaya, St-Peterbsurg 198504, Russia \\ ${ }^{4}$ Skolkovo Institute of Science and Technology Novaya Street, 100, Skolkovo 143025, Russian Federation \\ ${ }^{5}$ Department of Applied Mathematics and Theoretical Physics, University of Cambridge, \\ Cambridge, CB3 OWA, United Kingdom \\ (Received 19 December 2015; revised manuscript received 17 April 2016; published 26 August 2016)
}

\begin{abstract}
We investigate the phase coupling between spatially separated polariton condensates under nonresonant optical pulsed excitation. In the simple case of two condensates, we observe phase locking either in symmetric or antisymmetric states. We demonstrate that the coupling symmetry depends both on the separation distance and outflow velocity from the condensates. We interpret the observations through stimulated relaxation of polaritons to the phase configuration with the highest occupation. We derive an analytic criterion for the phase locking of a pair-polariton condensate and extend it to polariton multiplets. In the case of three condensates, we predict theoretically and observe experimentally either in-phase locking or the appearance of phase winding with phase differences of $\pm 2 \pi / 3$ between neighbors. The latter state corresponds to a vortex of winding number \pm 1 across the three polariton condensates.
\end{abstract}

DOI: 10.1103/PhysRevX.6.031032

Subject Areas: Condensed Matter Physics,

Nonlinear Dynamics,

Semiconductor Physics

\section{INTRODUCTION}

In semiconductor microcavities in the strong-coupling regime, cavity photons and intracavity excitons mix to a breed of bosonic optoelectronic excitations, called polaritons $[1,2]$. Below the temperature of quantum degeneracy, polaritons undergo a thermodynamic phase transition wherein phase correlations spontaneously occur and build up to a macroscopic coherent state [3,4]. This process is an example of spontaneous symmetry breaking in a many-body system [5-7]. Polaritons benefit from the low effective mass of cavity photons that enables hightemperature condensation, and the strongly interacting nature of excitons, rendering polariton condensates a promising test bed for many-body quantum technologies. Unlike atomic Bose-Einstein condensates [8,9], polaritons can condense out of thermal equilibrium [10]. Polariton condensates have been realized in several material systems under optical excitation including $\mathrm{CdTe}$, GaAs, GaN, $\mathrm{ZnO}$, and organic semiconductors [11-16], while most recently,

\footnotetext{
*Corresponding author. n.g.berloff@damtp.cam.ac.uk

Corresponding author. pavlos.lagoudakis@soton.ac.uk
}

Published by the American Physical Society under the terms of the Creative Commons Attribution 3.0 License. Further distribution of this work must maintain attribution to the author(s) and the published article's title, journal citation, and DOI. polariton condensation was observed under electrical injection [17]. Upon condensation, polaritons experience an increase in in-plane momentum due to their repulsion from hot excitons and other polaritons $[18,19]$. In the case of multiple, spatially separated condensates, out-flowing polaritons result in coupling across the multiplet of condensates.

In this article, we investigate the mechanism of coupling between spatially separated polariton condensates starting from a simple two-condensate configuration. We study the dependence of the phase configuration of the condensate pair upon their separation and outflow velocities and show that they phase-lock either in symmetric or antisymmetric states. We interpret the observation through stimulated relaxation of polaritons to the phase configuration with the highest polariton occupation. Previously, the coupling mechanism was attributed to the coherent "ballistic coupling" mechanism, whereby each condensate center is resonantly pumped by the outflow from the neighboring condensates, causing all condensates to be locked in phase $[20,21]$, and antisymmetric states were interpreted by one of us (N.G.B.) as a higher energy state resulting from the initial system noise. We generalize our results to any lattice geometry showing how stimulated relaxation could result in macroscopic states, where all condensates are either inphase or in nontrivial phase configurations. We test the latter case in an equidistant triangle of three condensates, where nearest neighbors are predicted to have $\pm 2 \pi / 3$ phase difference. We imprint an equidistant triangle of polariton 
condensates, and by varying the length of the edges, we observe a transition from a trivial all-in-phase configuration to $\pm 2 \pi / 3$ phase difference between nearest neighbors. The latter configuration corresponds to the simplest case of a vortex across multiple sites of a polariton condensate lattice.

The article is organized as follows: In Sec. II, we describe the sample and experimental configuration, investigate the dynamics of a pair-polariton condensate, and provide a phenomenological interpretation to the observed phenomena. In Sec. III, we derive an analytic criterion for the phase locking of two adjacent condensates and generalize the findings to three or more condensates. In Sec. IV, we test our prediction on three coupled condensates and demonstrate lattice-vortex formation. We conclude in Sec. V.

\section{POLARITON DYADS}

The semiconductor microcavity structure studied here was grown by molecular beam epitaxy and consists of a $\lambda / 2$ AlAs cavity embedded between two $\mathrm{Al}_{0.2} \mathrm{Ga}_{0.8} \mathrm{As} / \mathrm{AlAs}$ distributed Bragg reflectors (DBRs) with 16 (top) and 20 (bottom) pairs, respectively. Three sets of 7-nm quantum wells (QWs) are embedded in the antinode of the electric field in the cavity and the first antinodes in the DBRs, respectively. This technique results in strong light-matter interactions at minimal cost of the cavity volume. The quality factor of the structure is around 2000 [22]. In this structure, the dynamics of polariton condensates have previously been studied under pulsed excitation [23]. The excitation conditions in the experiments described in this article are chosen so that the system is always in the strong-coupling regime. A detailed study of the dynamics of the transition from the strong- to the weak-coupling regime has been studied elsewhere [7,24].

The sample is cooled to about $10 \mathrm{~K}$ using a cold-finger cryostat. We excite the sample nonresonantly into the first Bragg mode (about $0.1 \mathrm{eV}$ above the cavity mode) at $\lambda \simeq$ $730 \mathrm{~nm}$ using an $80-\mathrm{MHz}$ train of 180-fs Fourier-limited pulses. The spatial profile of the excitation beam is modulated to two or three equal-sized Gaussian excitation spots using a reflective spatial light modulator (SLM). We use a high numerical aperture microscope objective $(\mathrm{NA}=0.7)$ to focus the spatially modulated beam to about $1.3 \mu \mathrm{m}$ in diameter at full-width-at-half-maximum (FWHM) excitation spots. Light emitted from the sample is collected in reflection geometry through the same microscope objective. Real- and Fourier-space (dispersion) imaging is performed by projecting the corresponding image plane to an imaging camera with submicron optical resolution. Spectral real- and Fourier-space tomography is performed by scanning the corresponding image at the slit of a spectrophotometer coupled with an imaging camera with $50-\mu \mathrm{eV}$ energy resolution. Time-resolved real- and Fourier-space imaging is performed by scanning the corresponding image at the slit of a streak camera with 2-ps time resolution. Interference patterns between adjacent condensates are measured using a stabilized Michelson interferometer with one mirror replaced by a retro-reflector. Time-resolved interferometry is performed by scanning the interferogram at the slit of the streak camera.

We study the dynamics of phase locking between two neighboring polariton condensates in the aforementioned GaAs-based microcavity under nonresonant optical pulsed excitation. Above excitation threshold, two polariton condensates are formed with each excitation pulse. We use the SLM to control the distance between the two Gaussian excitation beams, while the excitation spot size remains unchanged. The distance between the two spots is kept within the range where the two condensates are always synchronized. When the two condensates are phase locked, we refer to them as a polariton dyad. The upper row of Fig. 1 shows the time-integrated real-space photoluminescence just above threshold for a polariton dyad at three different spatial separations. The intensity pattern resembles the interference of two phase-locked cylindrical waves with wave vectors equal to the in-plane wave vector of two resonant freely propagating polariton states. The interference pattern indicates that the two condensates are phase

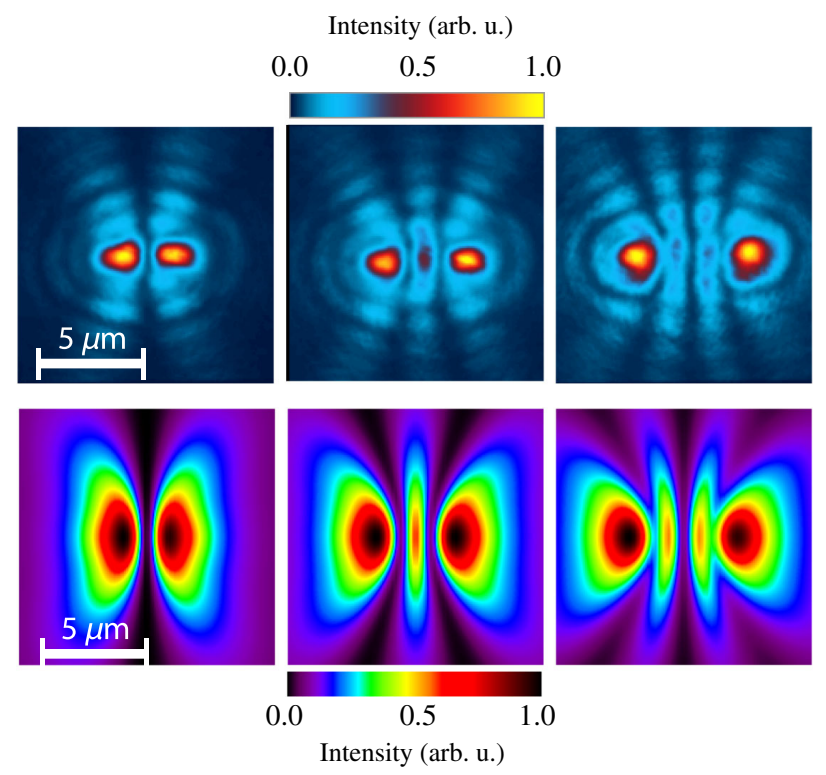

FIG. 1. (Upper row) The time-integrated images of two polariton condensates in real space at three different spatial separation distances. The condensates are formed simultaneously at $P \simeq P_{\text {th }}$, where $P_{\text {th }}$ is the threshold power for condensation. The observation of an interference pattern between the two condensates indicates that they are phase correlated. Zero intensity of the interference pattern in the middle of the two sources indicates that the two condensates are antisynchronized. (Lower row) Time-integrated results of numerical simulations of the Ginzburg-Landau equations with random initial phase averaged over 75 realizations at separation distances between the condensates as in the experiment above. The condensates phase lock, on average, to zero or $\pi$ phase difference depending on their spatial separation distance. 


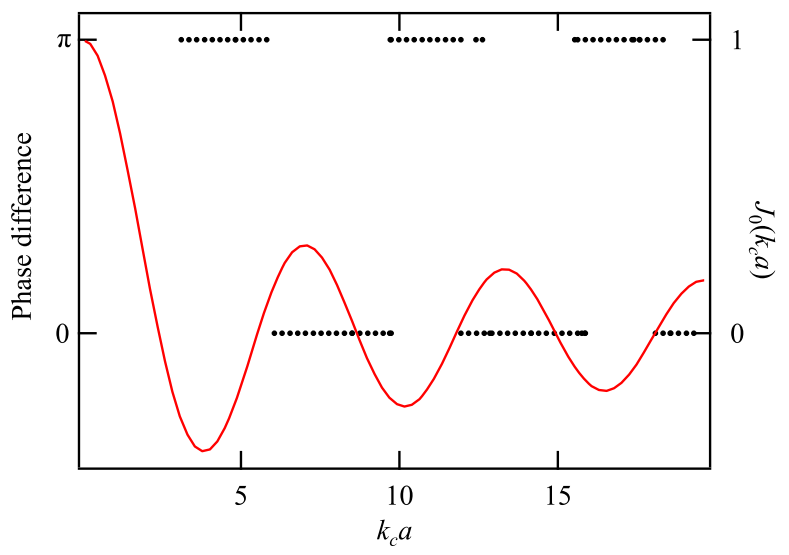

FIG. 2. The condensates flip from antisymmetric ( $\pi$ phase difference) to symmetric states (zero phase difference), shown with black dots as a function of the product of the outflow wave vector $k_{c}$ and the separation distance $a$. Here, we change $a$, whereas $k_{c}$ remains virtually unchanged. The solid red curve is the Bessel function calculated versus the dimensionless parameter $k_{c} a$.

locked with zero or $\pi$ phase difference depending on their separation. Similar to the case of two cylindrical waves, zero intensity of the interference pattern in the middle of the two sources indicates that the two condensates are in antiphase ( $\pi$ phase difference).

Figure 2 shows the phase difference, zero or $\pi$, for an extended range of separation distances produced by the SLM multiplied by the measured wave number $k_{c}$ of polaritons emitted by the condensates. The phase difference switches abruptly between in-phase and antiphase synchronization with a nearly periodic dependence on the product of the dyad's separation distance and the wave number $k_{c}$. In Fig. 3 we plot both the real- and Fourier-space photoluminescence images for four different separation distances. We note that by changing the separation distance of the pair, the condensate energy and wave vector remain virtually unchanged. Under these conditions, the periodicity of the in-phase or antiphase configurations is a function of the separation distance. The abrupt phase jumps as a function of the separation distance is atypical for the conventional Josephson coupling [25]. This is opposite to what we observe experimentally (and also numerically by solving the Ginzburg-Landau equation with 2D Langevin noise, as will be shown later).

The phase-locking mechanism can be phenomenologically understood when considering polariton condensation as an inherently symmetry-breaking process driven by stimulated relaxation into the state with the highest occupation number. Under nonresonant optical excitation, the phase of the pumping source is irrelevant and polaritons are initially created with random phases uniformly distributed across the pumping area. Injected polaritons spatially spread out because of the repulsive polariton-polariton interactions and, more importantly, because of their repulsive interactions with the exciton reservoir [26]. During the gradual increase of

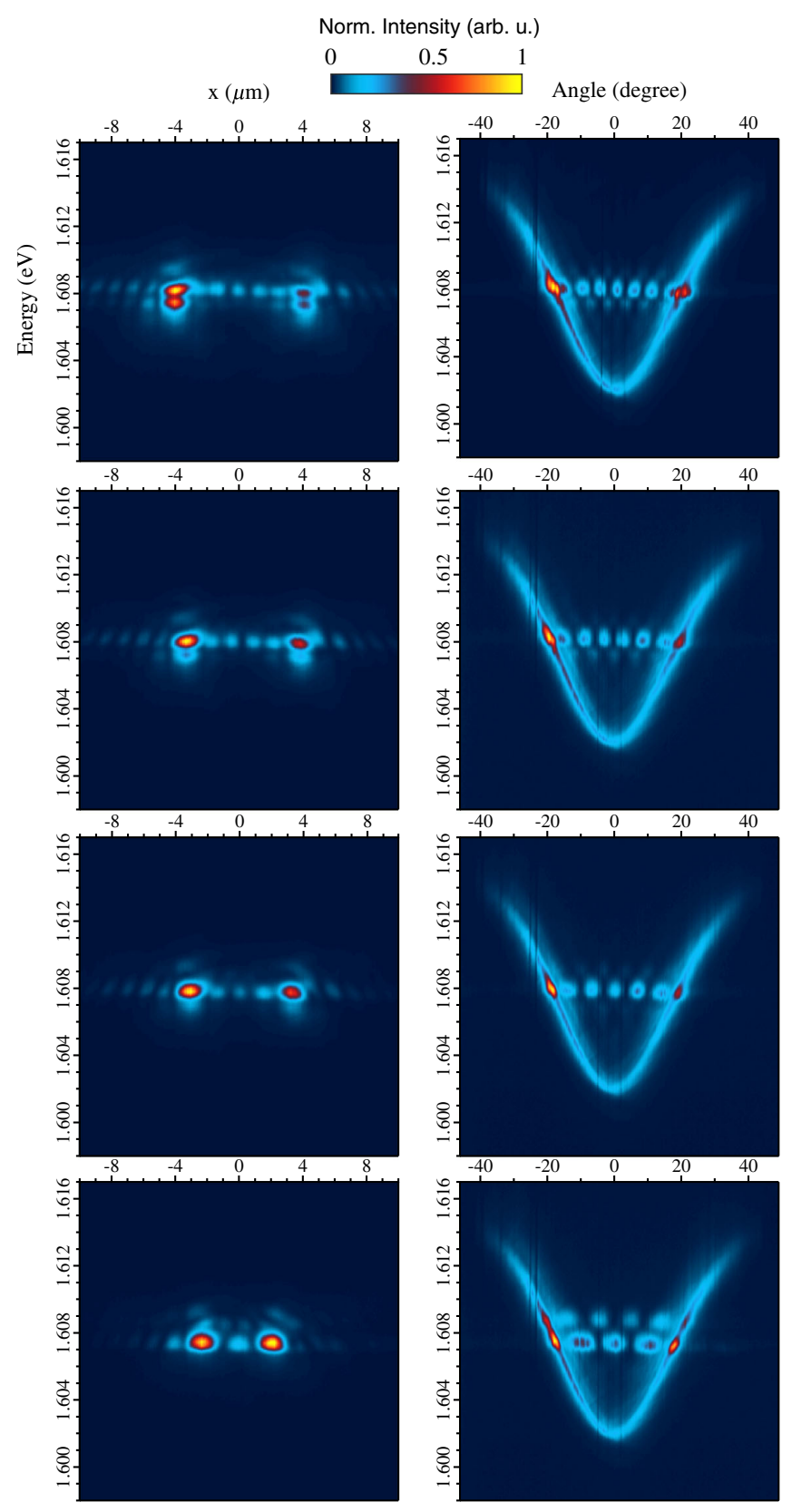

FIG. 3. Energy-resolved real- and Fourier-space images of two condensates are shown for different separation distances. The fringes in between the condensates are at the same energy with the condensates.

the polariton occupation, the phase configuration across the polariton dyad that carries the highest number of particles will reach condensation threshold first.

The type of interference (constructive or destructive) of the laterally out-flowing polaritons across the edge connecting the two pumping spots depends on the phase gained during the flow from one pumping spot to another. Thus, the type of interference should depend both on the separation distance between the two spots and on the in-plane wave vector of the out-flowing polaritons $k_{c}$. To 
investigate the dependence of the phase-locking mechanism on the in-plane wave vector $k_{c}$, we fix the separation distance between the two spots and increase the excitation density nearly twice above threshold. Under the pulsed excitation, the density of polaritons is transiently being reduced because of the losses and so is the in-plane wave vector $k_{c}$ of out-flowing polaritons. The transient nature of this experiment allows for condensation of the polariton dyad with fixed separation distance and varying (decreasing) in time in-plane wave vector $k_{c}$. We perform timeintegrated Fourier imaging of the polariton dyad and observe condensation at two discrete energy states as shown in Fig. 4(a). The arrows annotate the time frame during which the respective states occur, as measured from
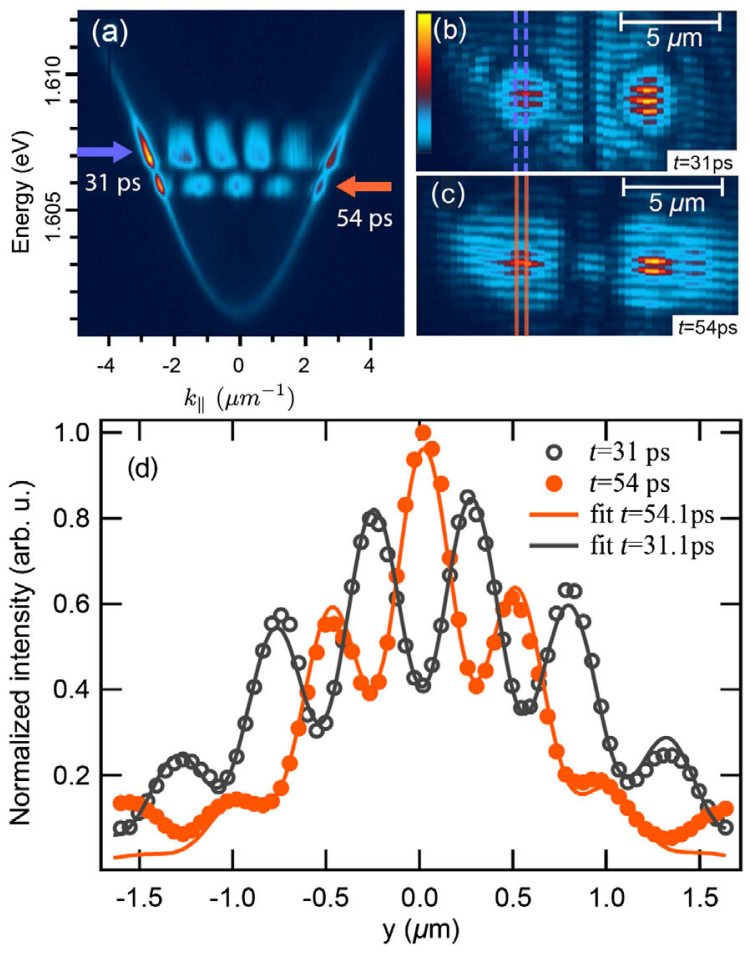

FIG. 4. (a) The time-integrated momentum space shows that condensation occurs at two distinctly different wave vectors. At $t=31 \mathrm{ps}$ (marked by a blue arrow), the polariton dyad is in antiphase synchronization. At $t=54 \mathrm{ps}$ (marked by an orange arrow), the polariton dyad is in in-phase synchronization. (b,c) Time-resolved interferograms at $t=31 \mathrm{ps}$ and $t=54 \mathrm{ps}$, respectively, showing the phase synchronization of the two condensates. The real-space scale bars in $(b, c)$ apply to both horizontal and vertical directions. The color scale in $(a-c)$ is shown as an inset bar in (b). The data in (a,b,c) are normalized. (d) The intensity profiles of the interferograms at the positions marked by the vertical lines in (b) (dashed blue) and (c) (solid orange) provide a direct experimental observation of the pi phase difference in the two synchronization regimes. The solid lines are the fits of a Gaussian function convoluted with a $\cos (k y+\phi)$, giving a phase difference of $(0.98 \pm 0.01) \times \pi$ between the two states. The zero of the horizontal axis is defined at the maximum of the intensity profile extracted from (c). time-resolved measurements. We note here the abrupt switching between the two states.

Notwithstanding that the symmetry flipping between the two successive states is evident by the even or odd number of nodes observed in the Fourier image of Fig. 4(a), we perform time-resolved spatial interferometry and directly measure their relative phases. We use an actively stabilized Michelson interferometer in a mirror-retroreflector configuration to interfere with the emission of the two spots. Figure 4(b) shows the interference fringes at the onset of condensation (31 ps after excitation), where the condensates are in antiphase. As time evolves, the two condensates desynchronize (loss of fringe visibility) and resynchronize at a later time (54 ps) to an in-phase configuration, as shown in Fig. 4(c). Figure 4(d) shows the $\pi$ phase jump between the two states from the intensity profiles extracted at the blue and orange lines of Figs. 4(b) and 4(c). We have therefore shown that the type of interference we observe also depends on the wave vector of the outflowing polaritons.

In the next section, we analyze the governing equations to evaluate the in-plane wave vector of outflowing polaritons $k_{c}$ in terms of the system and pumping parameters and arrive at a criterion for phase differences between two or more condensates, namely, maximization of the total number of polaritons in the condensates. This criterion provides a good agreement with the experimental observations.

\section{THEORY OF PHASE LOCKING ACROSS A LATTICE CONDENSATE}

To describe the behavior of the coupled condensates, we use the Ginzburg-Landau equation [27-29]:

$$
\begin{aligned}
i \hbar \frac{\partial \psi(\mathbf{r})}{\partial t}= & \left\{-\frac{\hbar^{2}}{2 m} \nabla_{\mathbf{r}}^{2}+\frac{i \hbar}{2}\left[R_{R} n_{R}(\mathbf{r})-\gamma_{C}\right]\right. \\
& \left.+\hbar g|\psi(\mathbf{r})|^{2}+\hbar \mathcal{G} P(\mathbf{r})\right\} \psi(\mathbf{r})+\hbar f(\mathbf{r}, t),
\end{aligned}
$$

where $\psi$ is the condensate wave function, $m$ is the effective polariton mass, $R_{R}$ is the incoming rate of polaritons from a hot exciton reservoir with a local density $n_{R}$ to the condensate, $\gamma_{C}$ is the decay rate of polaritons, $g$ is the repulsive polariton-polariton interaction (pseudo)potential, $P$ is the spatially dependent pumping rate of excitons in the reservoir, and $\mathcal{G}$ represents repulsive interactions of the condensate with the pump. Similar to Refs. [30,31], $f(\mathbf{r}, t)$ is the Langevin noise given by the correlator

$$
\left\langle f(\mathbf{r}, t) f^{*}\left(\mathbf{r}^{\prime}, t^{\prime}\right)\right\rangle=R_{R} n_{R}(\mathbf{r}) \delta\left(t-t^{\prime}\right) \delta\left(\mathbf{r}-\mathbf{r}^{\prime}\right) .
$$

Equation (1) is coupled to a rate equation for the reservoir, given by

$$
\dot{n}_{R}(\mathbf{r})=P(\mathbf{r})-\gamma_{R} n_{R}(\mathbf{r})-R_{R} n_{R}(\mathbf{r})|\psi|^{2},
$$


where the diffusive transport of hot excitons is neglected. The total number of condensed polaritons is

$$
I=\int|\psi(\mathbf{r})|^{2} d \mathbf{r} .
$$

During the buildup of the polariton occupancy and in the process of formation of coherence, the system will condense into the state that has the maximum total number density $I$.

To get the large distance behavior of a single condensate under a radially symmetric pump $P(r)$ with $\max _{r} P(r)=P(0) \equiv P_{\max }$, we consider the steady state of Eqs. (1) and (3): $\psi=\Psi(r) \exp [-i \mu t / \hbar]$ and $n_{R}=P(r) /\left(\gamma_{R}+R_{R}|\Psi|^{2}\right)$, where $\mu$ is the polariton energy that characterizes this steady state. With the Madelung transformation $\Psi=\sqrt{\rho(r)} \exp [i S(r)]$ for the number density $\rho=|\Psi|^{2}$ and phase $S$, the real and imaginary parts of Eq. (1) lead to the mass continuity and the integrated form of the Bernoulli equation:

$$
\begin{gathered}
\nabla \cdot(\rho \mathbf{u})=\left[\frac{R_{R} P(r)}{\gamma_{R}+R_{R} \rho(r)}-\gamma_{C}\right] \rho(r), \\
\mu=-\frac{\hbar^{2}}{2 m} \frac{\nabla^{2} \sqrt{\rho}}{\sqrt{\rho}}-\frac{m u^{2}}{2}+\hbar g \rho+\hbar \mathcal{G P}(r),
\end{gathered}
$$

where the velocity $\mathbf{u}=(\hbar / m) \nabla S(r)$ and $u=|\mathbf{u}|$. From Eq. (6), it follows that $u \approx \sqrt{2 \mu / m}$ away from the pumping region. The polariton energy $\mu$ can be approximated by assuming a sufficiently large pumping spot so that the quantum pressure term $\left(\hbar^{2} / 2 m\right) \nabla^{2} \sqrt{\rho} / \sqrt{\rho}$ and $u^{\prime}(0)$ can be neglected in view of the slow variation of $u$ and $\rho$ at the center of the pumping area. From Eq. (5), we estimate

$$
\rho(0) \approx \frac{P_{\max }}{\gamma_{C}}-\frac{\gamma_{R}}{R_{R}},
$$

and, therefore, from Eq. (6)

$$
\mu \approx \frac{\hbar g}{\gamma_{C}} P_{\max }-\frac{\hbar g \gamma_{R}}{R_{R}}+\hbar \mathcal{G} P_{\max } .
$$

From the parameters of the experiment, the second term is negligible in comparison with the last term which dominates. Away from the pumping spot, the wave function of a single condensate can be approximated by

$$
\Psi(r)=\sqrt{\rho(r)} \exp [i \sqrt{2 \mu m} r / \hbar],
$$

where an arbitrary constant phase can be added to the argument of the exponent. By this, we identify the outflow wave number $k_{c}=\sqrt{2 \mu m} / \hbar$, with $\mu$ given in Eq. (8). We approximate the wave function of two condensates of equal size located at $\pm \mathbf{a} / 2$ by the sum of the individual wave functions

$$
\tilde{\Psi}(\mathbf{r})=\left[\Psi\left(\mathbf{r}+\frac{\mathbf{a}}{2}\right)+e^{i \theta} \Psi\left(\mathbf{r}-\frac{\mathbf{a}}{2}\right)\right]
$$

where $\theta$ is the phase difference between the two condensates. The total number of condensed polaritons, Eq. (4), becomes

$$
I=\int|\tilde{\Psi}(\mathbf{r})|^{2} d \mathbf{r}=\int \frac{d \mathbf{k}}{(2 \pi)^{2}}|\hat{\Psi}(\mathbf{k})|^{2},
$$

with the Fourier transform

$$
\hat{\Psi}(\mathbf{k})=\int d \mathbf{r} e^{-i \mathbf{k} \cdot \mathbf{r}} \tilde{\Psi}(\mathbf{r})=\hat{\Psi}(k)\left[e^{i \mathbf{k} \cdot(\mathbf{a} / 2)}+e^{i \theta} e^{-i \mathbf{k} \cdot(\mathbf{a} / 2)}\right],
$$

where $\hat{\Psi}(k)=2 \pi \int_{0}^{\infty} \sqrt{\rho(r)} \exp \left[i k_{c} r\right] J_{0}(k r) r d r$, and $J_{0}$ is the Bessel function. The total number of condensed polaritons can then be written

$I=2 \int \rho(\mathbf{r}) d \mathbf{r}+\frac{1}{2 \pi^{2}} \int|\hat{\Psi}(k)|^{2} \cos (\mathbf{k} \cdot \mathbf{a}-\theta) d \mathbf{k}$.

An analytical progress can be made by assuming that the dimensionless quantity $k_{c} L \gg 1$, where $L$ is the characteristic width of the condensate density. Under this assumption, $|\hat{\Psi}(k)|^{2} \approx\left|\hat{\Psi}\left(k_{c}\right)\right|^{2} \delta\left(k-k_{c}\right)$. This assumption is supported by the direct experimental measurement of $|\hat{\Psi}(k)|^{2}$, which is highly localized (for instance, see the right panel of Fig. 3). Substituting this in Eq. (13) and using a symmetry argument gives the total number of condensed polaritons as

$$
\begin{aligned}
I & =2 \int \rho(\mathbf{r}) d \mathbf{r}+\frac{\left|\hat{\Psi}\left(k_{c}\right)\right|^{2}}{2 \pi^{2}} \cos \theta \int \delta\left(k-k_{c}\right) \cos (\mathbf{k} \cdot \mathbf{a}) d \mathbf{k} \\
& =2 \int \rho(\mathbf{r}) d \mathbf{r}+A J_{0}\left(k_{c} a\right) \cos \theta
\end{aligned}
$$

where $A=k_{c}\left|\hat{\Psi}\left(k_{c}\right)\right|^{2} / \pi>0$ depends on the details of the system parameters. For two condensates, $I$ is maximized for in-phase coupling if $J_{0}\left(k_{c} a\right)>0$ and for antiphase coupling if $J_{0}\left(k_{c} a\right)<0$. The relation (14) defines the dependence of the total number of condensed polaritons on the phase difference between the individual condensates. In the process of condensate formation, the phase symmetry is spontaneously broken to a state that maximizes the total occupation number $I$. At each separation, the condensates pick the state with maximum density and flip between in-phase and antiphase states in a nearly periodic fashion as their separation changes according to the sign of $J_{0}\left(k_{c} a\right)$, which agrees with the experimental results depicted in Fig. 2 together with $J_{0}\left(k_{c} a\right)$. In the Appendix, we analyze the limits of applicability of the main 
assumption used in identifying the switching between phases with zeros of $J_{0}\left(k_{c} a\right)$ and show that the slight deviation of this criterion from the experimental data seen in Fig. 2 is connected to $k_{c} L$ not being sufficiently large.

Furthermore, we numerically integrate Eq. (1) using a fifthorder Adams-Bashforth-Moulton predictor-corrector method and the following parameters: $\hbar R_{R}=0.05 \mu \mathrm{m}^{2} \mathrm{meV}$, $\mathcal{G}=0.0175 \mu \mathrm{m}^{2}, \hbar g=0.02 \mathrm{meV} \mu \mathrm{m}^{2}, \gamma_{C}=0.5 \mathrm{ps}^{-1}$, and $\gamma_{R}=0.01 \mathrm{ps}^{-1} . P$ is set to give the observed blueshift in the experiment. The lower panel in Fig. 1 shows simulations of the Ginzburg-Landau equation with random initial conditions, coupled to a hot exciton reservoir excited by the nonresonant pump [27]. Each figure is a time-integrated average of 75 realizations. The fringes between the condensates are, on average, due to interference between condensates that are phase locked in either zero or $\pi$ phase difference.

Generalizing Eq. (14) to $N$ condensates placed at positions $\mathbf{R}_{n}$ and having phases $\theta_{n}$, the total number of condensed polaritons is given by

$I=N \int \rho(\mathbf{r}) d \mathbf{r}+A \sum_{n<n^{\prime}} \cos \left(\theta_{n}-\theta_{n^{\prime}}\right) J_{0}\left(k_{c}\left|\mathbf{R}_{n}-\mathbf{R}_{n^{\prime}}\right|\right)$,

where the summation is over all $N(N-1) / 2$ pairs. In the case of an equilateral triangular lattice, $I$ is maximized when either all three neighbors are in phase for $J_{0}\left(k_{c} a\right)>0$ or when there is a $2 \pi / 3$ phase difference between the neighbors for $J\left(k_{c} a\right)<0$. The nontrivial case where there is a $2 \pi / 3$ phase difference going clockwise or anticlockwise between the neighbors corresponds to two topologically different global vortex states with a winding number of +1 or -1 .

In the next section, we elucidate the validity of the phasecoupling mechanism described here in the phase-locking dynamics of three neighboring condensates positioned at the vertices of an equilateral triangular lattice condensate.

\section{POLARITON TRIADS}

We modulate the spatial profile of the excitation beam to create three equally sized spots positioned at the vertices of an equilateral triangle using the same experimental configuration as in Sec. II. The spots are focused to about $1.3 \mu \mathrm{m}$ in diameter measured at FWHM. As in the case of polariton dyads, under nonresonant excitation, three polariton condensates are formed at the vertices of the excitation pattern, a polariton triad. Figures 5(a) and 5(b) show photoluminescence spectral tomography images for two different edge lengths of the excitation pattern. Observation of dark and bright fringes at the energy of the condensates indicates phase locking across the polariton triad. From the interference pattern, we can infer that constructive interference at the center of the triangle corresponds to the case where the polariton triad is in an all-in-phase configuration. The pattern of destructive interference at the center of the triangle corresponds to the case where the three condensates are in an out-of-phase configuration. The two phase configurations are reproduced from numerical simulations of Eqs. (1) and (3). Figures 5(c) and 5(d) show the results of numerical simulations of the condensate density averaged over 75 realizations with random initial phase conditions and pumping profile tuned to match the experimental condensate intensities. Examining the relationship between the maximum density and the phase configuration of the polariton triad, we find that the total number of condensed polaritons is maximized when either all three neighbors are in phase $\left[J_{0}\left(k_{c} a\right)>0\right]$ or when there is a $2 \pi / 3$ phase difference between neighbors $\left[J_{0}\left(k_{c} a\right)<0\right]$. Figure $5(\mathrm{c})$ corresponds to the all-in-phase configuration. In the nontrivial case of $2 \pi / 3$ phase difference between neighbors, the numerical simulations produce two topologically distinct degenerate states that correspond to clockwise and anticlockwise $2 \pi / 3$ phase difference between adjacent neighbors. The density profile of Fig. 5(d) is the average of these two states, shown in Figs. 5(e) and 5(f), respectively. Both states have near-zero density in the center of the polariton triad. These two states represent phase vortices across the polariton triad with winding numbers of +1 or -1 , shown in Fig. 5(g) and 5(h), respectively. The formation of a polariton triad with +1 or -1 winding number is stochastic and thus not accessible in the spectral tomography image of Fig. 5(b) that is the result of the average of about $10^{8}$ realizations.

In order to experimentally access the various phase configurations, we use time-resolved spatial interferometry, as in Sec. II, and interfere the emission of two neighbors. We choose an edge length that at condensation threshold results in the vortex phase configuration across the polariton triad. We increase the excitation density so as to allow for the next phase-locked state to occur, which corresponds to the all-in-phase configuration. We time resolve the photoluminescence pattern and observe the transition between the two states. This transition can also be observed through Fourier imaging (not shown here). We time resolve the interferogram and observe a dynamical transition from the all-in-phase state to the out-of-phase state. Figures 5(i) and 5(j) show the interferograms when the condensates are all-in-phase (i) and when they are out-of-phase types (j). Figure $5(\mathrm{k})$ shows the line profiles taken from these two interferograms that differ by about $2 \pi / 3$. In previous experimental work, under nonresonant excitation of three condensates, only the all-in-phase configuration was observed [21]. A vortex hexagonal lattice of alternating +1 and -1 vortices was predicted in Ref. [32]. In our experiments, we observe a vortex state winding across the polariton triad, which corresponds to the simplest vortex condensate lattice. 

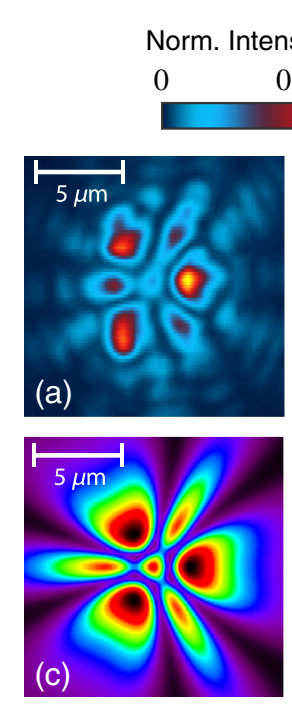
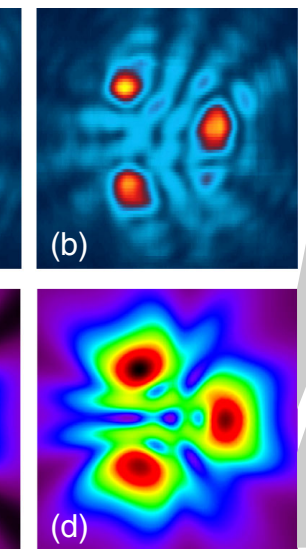
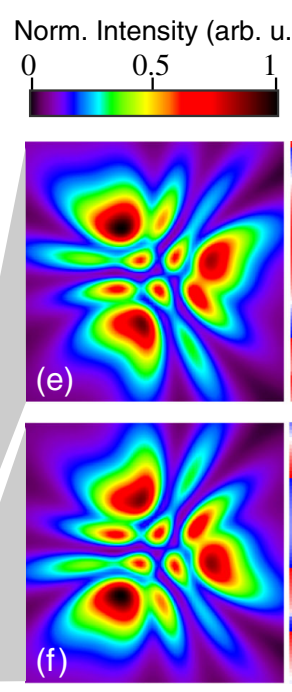

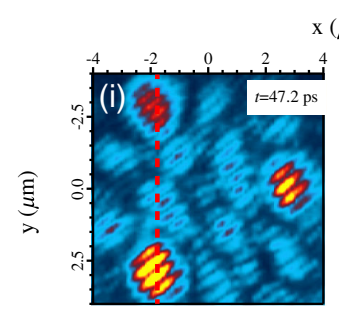

$\mathrm{x}(\mu \mathrm{m})$
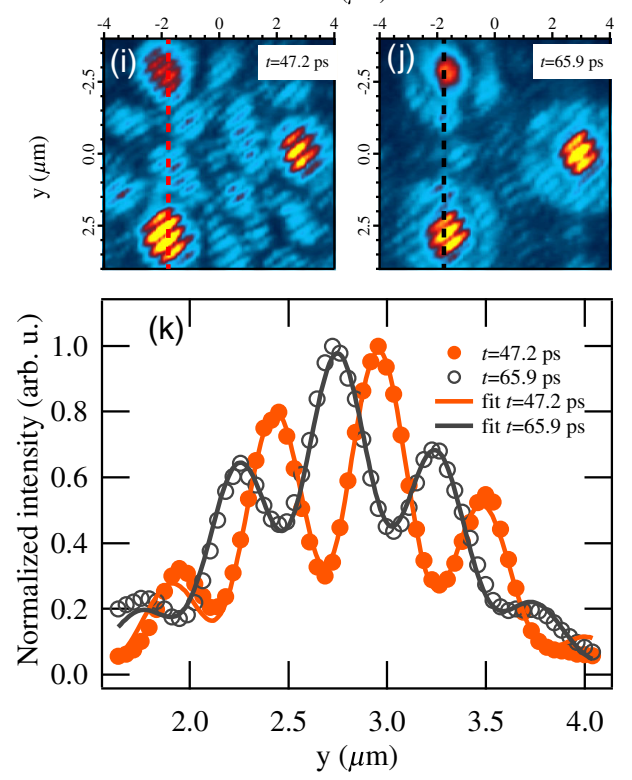

FIG. 5. (a-d) Time-integrated real-space tomography images of the triangular array condensates are shown for $a=4 \mu \mathrm{m}$ (a) and $a=5.5 \mu \mathrm{m}$ (b). The real-space scale bar of (a) also applies to (b). (c,d) Simulations of the Ginzburg-Landau equation with random initial phase, averaged over 75 realizations at different separations matching the experimental conditions are shown. In (c), all three condensates are in phase. In (d), we plot the average of vortices with \pm 1 winding numbers shown in (e) and (f). The phase diagrams of (e) and (f) are shown in (g) and (h), proving that they are indeed vortex states with winding numbers of $+1(\mathrm{~h})$ or $-1(\mathrm{~g})$. (i,j) The timeresolved interferograms resulting from the interference of neighboring condensates are shown at $t=47.2 \mathrm{ps}(\mathrm{i})$ and $t=65.9 \mathrm{ps}(\mathrm{j})$. The color bar of (a) and (b) applies to the data of (i) and (j). (k) The intensity profiles of the interferograms along the lines marked by the vertical lines in (i) (dashed red) and (j) (dashed black) provide a direct experimental observation of the nearly $2 \pi / 3$ phase difference in the two synchronization regimes. The line profiles of (i) and (j) taken at $x=-1.9 \mu \mathrm{m}$. The solid lines are the fits by the convolution of a Gaussian function with $\cos (k y+\phi)$ resulting in a $(0.78 \pm 0.02) \times \pi$ phase difference between the two fits.

\section{CONCLUSIONS}

In conclusion, we study the phase-coupling mechanism between spatially separated polariton condensates. We demonstrate experimentally that depending on the separation distance between condensates and the velocity of outflowing polaritons, two condensates synchronize with zero or $\pi$ phase difference. In the bottom-up process of polariton condensation, where the density is gradually increasing, condensation occurs at the phase configuration that carries the highest occupancy.

We derive the analytic criterion for the phase-locking configuration, and our simulations show how the locking into a particular phase difference is directly related to the spontaneous symmetry breaking and the nonlinearity at the condensation phase transition. In the simplest case of a polariton dyad, the phase-coupling mechanism causes the two condensates to couple in antiphase for $J_{0}\left(k_{c} a\right)<0$ and to couple in phase for $J_{0}\left(k_{c} a\right)>0$.

For three pumping spots, we theoretically predicted and experimentally demonstrated the formation of spontaneous global vorticity that corresponds to the phase winding among the pumping spots. The condensate array spontaneously picks a clockwise or anticlockwise rotating direction due to phase fluctuations at the onset of condensation.
Our observations demonstrate spontaneous symmetry breaking in real space in a dissipative bosonic system.

All data supporting this study are openly available from the University of Southampton repository at [33].

\section{ACKNOWLEDGMENTS}

The authors acknowledge the use of the IRIDIS High Performance Computing Facility, at the University of Southampton, and thank Jacqueline Bloch and Aristide Lemaitre for the provision of the sample. P.G.L. and A. V. K. acknowledge EPSRC through Programme Grant on Hybrid Polaritonics EP/M025330/1 and EP/F026455/1 for co-supporting this work. N.G. B acknowledges financial support by the Ministry of Education and Science of the Russian Federation 1425320 (Project DOI: RFMEFI58114X0006). Y. G. R. acknowledges financial support by CONACYT (Mexico) under Grant No. 251808.

\section{APPENDIX}

Here, we explore the applicability of the criterion for the switching between in-phase and out-phase regimes between two condensates that we derived in Sec. III. The main assumption made is that the density of the 

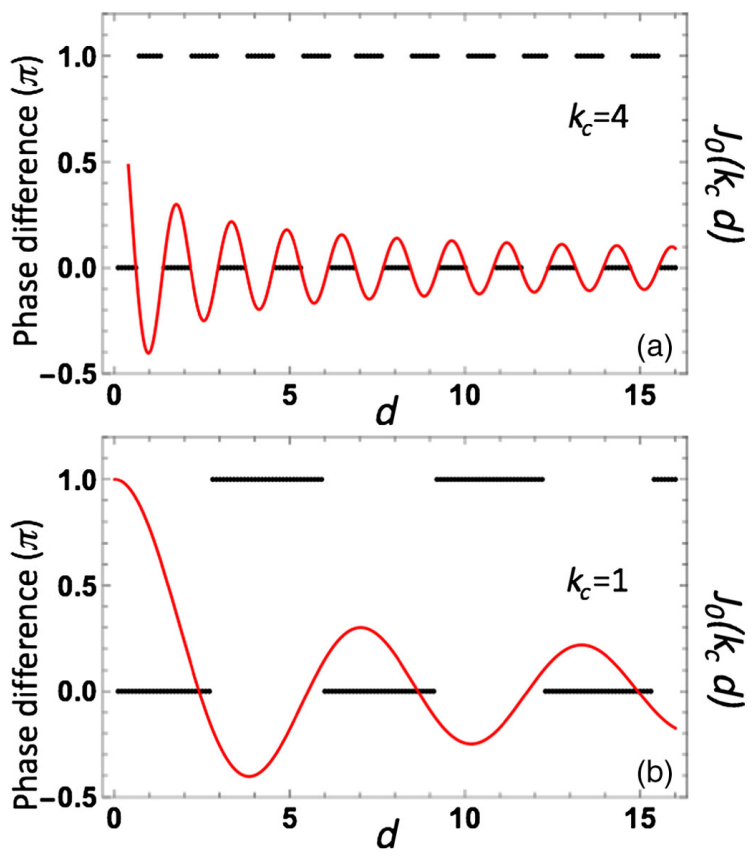

FIG. 6. The condensates flip from antisymmetric ( $\pi$ phase difference) to symmetric states (zero phase difference), shown with black segments as a function of the separation distance for two values of the outflow wave number (a) $k_{c}=4$ and (b) $k_{c}=1$. The solid red curves are the Bessel functions, $J_{0}\left(k_{c} d\right)$.

Hankel transform of the condensate wave function has a delta-function behavior $|\hat{\Psi}(k)|^{2} \approx\left|\hat{\Psi}\left(k_{c}\right)\right|^{2} \delta\left(k-k_{c}\right)$. This assumption is justified if $k_{c} L \gg 1$, where $L$ is the characteristic width of the condensate density and $k_{c}$ is the outflow wave number. To illustrate, we consider a trial wave function $\Psi(r)=\operatorname{sech}(q r) \exp \left[i k_{c} r\right]$, where the parameter $q$ defines the FWHM $L$ of the density $|\Psi|^{2}$ via $q L=1.76275$. We form two sums with in-phase and antiphase coupling of two condensates separated by distance $d$ that we denote as $\Psi_{+}$and $\Psi_{-}$, respectively:

$$
\Psi_{ \pm}=\Psi\left(\mathbf{r}+\frac{\mathbf{d}}{2}\right) \pm \Psi\left(\mathbf{r}-\frac{\mathbf{d}}{2}\right)
$$

We numerically integrate to find $I_{ \pm}=\int\left|\Psi_{ \pm}\right|^{2} d \mathbf{r}$ for two sets of data: $q=1 / 2$ and $k_{c}=4$ or $k_{c}=1$, which give $k_{c} L \approx 14$ and $k_{c} L \approx 3.5$, respectively. For each $d$, we choose the phase difference that maximizes $\left\{I_{+}, I_{-}\right\}$and plot it together with $J_{0}\left(k_{c} d\right)$. The resulting graphs are shown in Fig. 6. As seen from the graphs, the criterion for the switching according to the sign of $J_{0}\left(k_{c} d\right)$ is well satisfied for the case (a) with $k_{c} L \approx 14$. For $k_{c} L \approx 3.5$, there is some deviation from the criterion. From our experimental data, we get $k_{c} L \approx 4$, so it is closer to Fig. 6(b) with the same order of misalignment. We conclude, therefore, that our analytic criterion is accurate for $k_{c} L \gg 1$.
[1] C. Weisbuch, M. Nishioka, A. Ishikawa, and Y. Arakawa, Observation of the Coupled Exciton-Photon Mode Splitting in a Semiconductor Quantum Microcavity, Phys. Rev. Lett. 69, 3314 (1992).

[2] A. V. Kavokin, J. Baumberg, G. Malpuech, and F. P. Laussy, Microcavities (Oxford University Press, Oxford, 2007).

[3] J. Kasprzak, M. Richard, S. Kundermann, A. Baas, P. Jeambrun, J. M. J. Keeling, F. M. Marchetti, M. H. Szymanska, R. André, J. L. Staehli et al., Bose-Einstein Condensation of Exciton Polaritons, Nature (London) 443, 409 (2006).

[4] R. Balili, V. Hartwell, D. Snoke, L. Pfeiffer, and K. West, Bose-Einstein Condensation of Microcavity Polaritons in a Trap, Science 316, 1007 (2007).

[5] D. Snoke, Spontaneous Bose Coherence of Excitons and Polaritons, Science 298, 1368 (2002).

[6] J. J. Baumberg, A. Kavokin, S. Christopoulos, A. Grundy, R. Butté, G. Christmann, D. Solnyshkov, G. Malpuech, G. Baldassarri Höger von Högersthal, E. Feltin, J. F. Carlin, and N. Grandjean, Spontaneous Polarization Buildup in a Room-Temperature Polariton Laser, Phys. Rev. Lett. 101, 136409 (2008).

[7] H. Ohadi, E. Kammann, T. C. H. Liew, K. G. Lagoudakis, A. V. Kavokin, and P. G. Lagoudakis, Spontaneous Symmetry Breaking in a Polariton and Photon Laser, Phys. Rev. Lett. 109, 016404 (2012).

[8] M. H. Anderson, J. R. Ensher, M. R. Matthews, C. E. Wieman, and E. A. Cornell, Observation of Bose-Einstein Condensation in a Dilute Atomic Vapor, Science 269, 198 (1995).

[9] K. B. Davis, M. O. Mewes, M. R. Andrews, N. J. van Druten, D. S. Durfee, D. M. Kurn, and W. Ketterle, BoseEinstein Condensation in a Gas of Sodium Atoms, Phys. Rev. Lett. 75, 3969 (1995).

[10] J. Kasprzak, M. Richard, A. Baas, B. Deveaud, R. André, J.-Ph. Poizat, and Le Si Dang, Second-Order Time Correlations within a Polariton Bose-Einstein Condensate in a CdTe Microcavity, Phys. Rev. Lett. 100, 067402 (2008).

[11] L.S. Dang, D. Heger, R. André, F. Boeuf, and R. Romestain, Stimulation of Polariton Photoluminescence in Semiconductor Microcavity, Phys. Rev. Lett. 81, 3920 (1998).

[12] H. Deng, G. Weihs, C. Santori, J. Bloch, and Y. Yamamoto, Condensation of Semiconductor Microcavity Exciton Polaritons, Science 298, 199 (2002).

[13] S. Christopoulos, G. Baldassarri Höger von Högersthal, A. J. D. Grundy, P. G. Lagoudakis, A. V. Kavokin, J. J. Baumberg, G. Christmann, R. Butté, E. Feltin, J. F. Carlin, and N. Grandjean, Room-Temperature Polariton Lasing in Semiconductor Microcavities, Phys. Rev. Lett. 98, 126405 (2007).

[14] Feng Li, L. Orosz, O. Kamoun, S. Bouchoule, C. Brimont, P. Disseix, T. Guillet, X. Lafosse, M. Leroux, J. Leymarie et al., From Excitonic to Photonic Polariton Condensate in a ZnO-Based Microcavity, Phys. Rev. Lett. 110, 196406 (2013).

[15] K. S. Daskalakis, S. A. Maier, R. Murray, and S. Kéna-Cohen, Nonlinear Interactions in an Organic Polariton Condensate, Nat. Mater. 13, 271 (2014).

[16] J. D. Plumhof, T. Stöferle, L. Mai, U. Scherf, and R. F. Mahrt, Room-Temperature Bose-Einstein Condensation of 
Cavity Exciton-Polaritons in a Polymer, Nat. Mater. 13, 247 (2014).

[17] C. Schneider, A. Rahimi-Iman, N. Y. Kim, J. Fischer, I. G. Savenko, M. Amthor, M. Lermer, A. Wolf, L. Worschech, V. D. Kulakovskii et al., An Electrically Pumped Polariton Laser, Nature (London) 497, 348 (2013).

[18] M. Richard, J. Kasprzak, R. Romestain, R. André, and L. S. Dang, Spontaneous Coherent Phase Transition of Polaritons in CdTe Microcavities, Phys. Rev. Lett. 94, 187401 (2005).

[19] E. Wertz, L. Ferrier, D. D. Solnyshkov, R. Johne, D. Sanvitto, A. Lemaître, I. Sagnes, R. Grousson, A. V. Kavokin, P. Senellart, G. Malpuech, and J. Bloch, Spontaneous Formation and Optical Manipulation of Extended Polariton Condensates, Nat. Phys. 6, 860 (2010).

[20] G. Tosi, G. Christmann, N. G. Berloff, P. Tsotsis, T. Gao, Z. Hatzopoulos, P. G. Savvidis, and J. J. Baumberg, Sculpting Oscillators with Light within a Nonlinear Quantum Fluid, Nat. Phys. 8, 190 (2012).

[21] G. Tosi, G. Christmann, N. G. Berloff, P. Tsotsis, T. Gao, Z. Hatzopoulos, P. G. Savvidis, and J. J. Baumberg, Geometrically Locked Vortex Lattices in Semiconductor Quantum Fluids, Nat. Commun. 3, 1243 (2012).

[22] D. Bajoni, E. Semenova, A. Lemaître, S. Bouchoule, E. Wertz, P. Senellart, and J. Bloch, Polariton Light-Emitting Diode in a GaAs-Based Microcavity, Phys. Rev. B 77, 113303 (2008).

[23] M. Maragkou, A. J. D. Grundy, T. Ostatnický, and P. G. Lagoudakis, Longitudinal Optical Phonon Assisted Polariton Laser, Appl. Phys. Lett. 97, 111110 (2010).

[24] E. Kammann, H. Ohadi, M. Maragkou, A. V. Kavokin, and P. G. Lagoudakis, Crossover from Photon to ExcitonPolariton Lasing, New J. Phys. 14, 105003 (2012).
[25] K. G. Lagoudakis, B. Pietka, M. Wouters, R. André, and B. Deveaud-Pledran, Coherent Oscillations in an Exciton-Polariton Josephson Junction, Phys. Rev. Lett. 105, 120403 (2010).

[26] A. Askitopoulos, H. Ohadi, A. V. Kavokin, Z. Hatzopoulos, P. G. Savvidis, and P. G. Lagoudakis, Polariton Condensation in an Optically Induced Two-Dimensional Potential, Phys. Rev. B 88, 041308(R) (2013).

[27] M. Wouters, I. Carusotto, and C. Ciuti, Spatial and Spectral Shape of Inhomogeneous Nonequilibrium ExcitonPolariton Condensates, Phys. Rev. B 77, 115340 (2008).

[28] J. Keeling and N. G. Berloff, Spontaneous Rotating Vortex Lattices in a Pumped Decaying Condensate, Phys. Rev. Lett. 100, 250401 (2008).

[29] N. G. Berloff and J. Keeling, Universality in Modelling Non-equilibrium Pattern Formation in Polariton Condensates, in Physics of Quantum Fluids: New Trends and Hot Topics in Atomic and Polariton Condensates, edited by A. Bramati and M. Modugno (Springer-Verlag, Berlin, 2013).

[30] I. L. Aleiner, B. L. Altshuler, and Y. G. Rubo, Radiative Coupling and Weak Lasing of Exciton-Polariton Condensates, Phys. Rev. B 85, 121301(R) (2012).

[31] D. Read, T. C. H. Liew, Y. G. Rubo, and A. V. Kavokin, Stochastic Polarization Formation in Exciton-Polariton Bose-Einstein Condensates, Phys. Rev. B 80, 195309 (2009).

[32] J. Keeling and N. Berloff, Controllable Half-Vortex Lattices in an Incoherently Pumped Polariton Condensate, arXiv:1102.5302.

[33] http://eprints.soton.ac.uk/397772/. 